\title{
Kinematic analysis of preterm newborns' spontaneous movements for postural activity assessment
}

\author{
Jan Halek', Anita Muckova ${ }^{\mathrm{b}, \mathrm{c}}$, Zdenek Svobodac, Miroslav Janurac, Jana Marikova ${ }^{\mathrm{b}}$, Katerina Horakovad , Lumir Kantora, \\ Nina Nemcova ${ }^{d}$
}

\begin{abstract}
Aims. The objectives of this pilot study were to assess the potential use of 3D videography for analyzing the motion of the body center of mass (COM) in newborns and to determine differences in spontaneous movements between preterm and full-term infants.

Methods. The group comprised 10 preterm newborns (gestational age at birth between 26 and 37 weeks; birth weight 800 to $2960 \mathrm{~g}$; gestational age at the time of examination 34 to 39 weeks) and 10 full-term infants (gestational week 38 to 41 ; birth weight 2810 to $4360 \mathrm{~g}$ ). To determine the range of motion of the COM, 3D videography was used ( 2 cameras, $25 \mathrm{~Hz}$ ). When recording their movements, the infants were in the supine position, calm and awake. The recordings were processed using the APAS software. Selected points on the body were marked to obtain data for calculating the basic parameters of COM trajectories.

Results. The range of motion of the COM in both craniocaudal and anteroposterior directions was significantly greater in premature infants $(P<0.05$ and $P<0.01$, respectively) than in full-term babies. The variability of motion of the COM was significantly greater in the craniocaudal $(P<0.01)$ and anteroposterior $(P<0.05)$ directions in preterm babies. This was also valid for the velocity of motion of the COM in the craniocaudal direction $(P<0.05)$.

Conclusions. 3D videography can be used for experimental assessment of motor behavior in preterm infants. Basic kinematic characteristics of the motion of the COM (range, variability, velocity) are greater in preterm infants.

Key words: kinematic analysis, newborn, center of mass

Received: March 25, 2014; Accepted with revision: October 1, 2014; Available online: October 29, 2014 http://dx.doi.org/10.5507/bp.2014.053

${ }^{a}$ Department of Neonatology and Department of Pediatrics, University Hospital Olomouc and Faculty of Medicine and Dentistry, Palacky University Olomouc, Czech Republic

${ }^{b}$ Department of Physiotherapy, Faculty of Health Sciences, Palacky University Olomouc

'Department of Natural Sciences in Kinanthropology, Faculty of Physical Culture, Palacky University Olomouc

${ }^{d}$ Department of Rehabilitation, Faculty of Medicine, University of Ostrava

Corresponding author: Jan Halek, e-mail:jan.halek@fnol.cz
\end{abstract}

\section{INTRODUCTION}

A non-insignificant proportion of the newborn population is at risk for impaired development potentially affecting motor skills (cerebral palsy), as evidenced by the presence of risk factors. Significant risk factors include, in particular, preterm birth, certain congenital anomalies of the central nervous system (CNS) and hypoxic-ischemic encephalopathy. At present, no simple and objective method is available that would reliably predict development of such complications and allow early and targeted intervention in this at-risk population. To estimate further development, several methods from various fields may be used, such as results of CNS imaging (ultrasound or magnetic resonance imaging [MRI]), electrophysiological and clinical examinations. MRI of the brain is a valuable and objective tool; the drawbacks are high cost and the need for analgesia and sedation in young children. Its reliability in predicting the development of motor impairments is limited ${ }^{1}$. The problem in clinical neurological examination is the absence of standardized examinations to be used in infancy and the existence of numerous methods, some of which are strikingly different. The methods used are of various predictive value and depend on rater experience $^{2}$. Recently, assessment of the so-called general movement has proven promising. Recordings of movements are used to evaluate the quality of certain spontaneous general movements in infants and newborns ${ }^{3,4}$. Even with this time-consuming method, however, validity must be established in further studies ${ }^{5}$.

One approach used to analyze human motion is kinematic analysis. This group of methods includes the use of imaging techniques to identify the position of selected body points or segments and to record their movement in time and space. Subsequently, data are processed to provide quantitative and objective analyses of movement ${ }^{6,7}$.

The basic premise for voluntary motor activity in humans is to control the spatial position of the body and body segments. Development of this postural control is a very complex and comprehensive process influenced by numerous physiological and pathological factors ${ }^{8}$. Thus, assessing variability in postural control is one of the options for early detection of severe motor impairments.

The center of mass (COM) of the human body is defined as the point of the weight vector. It may be determined as the weighted average of positions of the COMs 
of individual body segments. Characteristics of the movement of the COM over time may be used to assess postural control ${ }^{9,10}$.

This pilot study aimed at verifying the potential use of kinematic analysis to characterize the movement of the COM in newborns and determining the difference between groups of preterm and full-term babies.

\section{MATERIALS AND METHODS}

\section{Patients}

The group comprised 20 newborns who were hospitalized in the Department of Neonatology of the University Hospital Olomouc at the time of investigation (Table 1). The experimental group included 10 preterm newborns with a gestational age at birth between 26 and 37 weeks and birth weight (BW) ranging from 800 to $2960 \mathrm{~g}$. At the time of examination, their gestational age was 34 to 39 weeks and their actual weight was 1930 to $2740 \mathrm{~g}$. The control group comprised 10 full-term newborns (gestational week 38 to 41 ) with a BW of 2810 to $4360 \mathrm{~g}$. The examination was performed on day 3 or later after birth. The inclusion criteria were good clinical condition, absence of severe complications (severe respiratory distress syndrome, bronchopulmonary dysplasia, severe infection complication, necrotising enterocolitis, periventricular leukomalacia, intra/periventricular hemorhage), congenital developmental anomalies and relevant medication (sedatives and analgetics etc.) (Table 1). Informed consent was obtained from parents of all patients and the study was approved by the local ethics committee.

\section{Equipment, measurement procedures}

Newborns' movements were recorded with two cameras (Sony DCR-TRV900E and Sony HDV 1080i, 25 Hz) synchronized with a light board with LEDs. Prior to each recording, the space was calibrated using a cuboid-shaped metal frame with marked points at predefined distances.
Before the video recording was started, the child was placed in the supine position on a disposable underpad in the recorded space. Children were one to two hours after feeding, the examination room was quit, dimly lighted and adequately heated $\left(22-25^{\circ} \mathrm{C}\right)$. When filmed, all children were awake and calm. Then, a 30-second recording was made of the infant's spontaneous movements in this position.

Subsequently, the recordings were processed using the APAS software (Ariel Dynamics. Inc., Trabuco Canyon, CA, USA). After synchronization of two corresponding recordings made with both cameras, selected anatomical points (the nose, centers of the pinnae, centers of the shoulder joints, medial humeral epicondyles, centers of the wrist, navel, xiphoid process, centers of the hips, medial femoral epicondyles and medial ankles) were marked in each picture.

With the APAS software, time-dependent trajectories of the markers in recordings were generated and smoothed using a quintic spline. To calculate the COM, first, COMs of the following individual segments were determined: trunk+head, right/left thigh, shank+foot, arm and forearm+hand. The resulting COM was calculated using relative segment masses ${ }^{11}$ and the formula: $\mathrm{x}_{\mathrm{COM}}=$ $\sum m_{i} \cdot x_{i}$, where $m_{i}$ is relative segment masses and $x_{i}$ is the $\mathrm{x}$-axes of COM of individual segments. Similarly, $\mathrm{y}_{\mathrm{COM}}$ and $\mathrm{z}_{\mathrm{COM}}$ were calculated.

\section{Measured parameters}

To allow comparison of the two groups, the following parameters were determined: the total range of motion of the COM - the percentage of the range of motion of a point relative to the infant's body length; variability of the COM motion - the standard deviation of motion of a point relative to the body length; and velocity of the COM motion - the mean velocity of a point relative to the body length. All parameters were determined both for individual movement directions (craniocaudal, antero-

Table 1. Experimental and control group profiles.

\begin{tabular}{|c|c|c|c|c|}
\hline & Sex & $\begin{array}{c}\text { Gestational age } \\
\text { (weeks) }\end{array}$ & $\begin{array}{l}\text { Birth weight } \\
\text { (g) }\end{array}$ & $\begin{array}{c}\text { Postnatal age } \\
\text { (days) }\end{array}$ \\
\hline Experimental group & $\mathrm{F}$ & 35 & 2140 & 18 \\
\hline \multirow{9}{*}{$(n=10)$} & M & 36 & 2960 & 3 \\
\hline & $\mathrm{F}$ & 35 & 2340 & 18 \\
\hline & M & 26 & 800 & 72 \\
\hline & M & 35 & 1490 & 22 \\
\hline & M & 27 & 980 & 63 \\
\hline & M & 31 & 1680 & 20 \\
\hline & $\mathrm{F}$ & 35 & 1930 & 17 \\
\hline & $\mathrm{F}$ & 31 & 1940 & 20 \\
\hline & M & 35 & 2840 & 3 \\
\hline Control group & $\mathrm{F}$ & 40 & 3570 & 3 \\
\hline \multirow{9}{*}{$(\mathrm{n}=10)$} & $\mathrm{F}$ & 41 & 3090 & 4 \\
\hline & $\mathrm{F}$ & 39 & 3390 & 3 \\
\hline & M & 39 & 2810 & 3 \\
\hline & $\mathrm{F}$ & 38 & 3370 & 4 \\
\hline & M & 41 & 4360 & 3 \\
\hline & M & 39 & 3430 & 3 \\
\hline & M & 38 & 3080 & 3 \\
\hline & M & 38 & 3090 & 3 \\
\hline & M & 41 & 3850 & 4 \\
\hline
\end{tabular}


Table 2. Basic statistical characteristics of measured COM parameters.

\begin{tabular}{|c|c|c|c|c|c|c|c|}
\hline \multirow{2}{*}{ Plane } & \multirow{2}{*}{ Infants } & \multicolumn{2}{|c|}{ Range } & \multicolumn{2}{|c|}{ Variability } & \multicolumn{2}{|c|}{ Velocity } \\
\hline & & Mean \pm SD & $P$ & Mean \pm SD & $P$ & Mean \pm SD & $P$ \\
\hline \multirow[t]{2}{*}{ Craniocaudal } & Preterm & $10.0 \pm 7.7$ & 0.017 & $2.0 \pm 0.9$ & 0.008 & $1.7 \pm 1.1$ & 0.028 \\
\hline & Full-term & $4.8 \pm 1.9$ & & $1.0 \pm 0.4$ & & $0.8 \pm 0.6$ & \\
\hline \multirow[t]{2}{*}{ Anteroposterior } & Preterm & $5.3 \pm 3.4$ & 0.006 & $1.1 \pm 0.4$ & 0.017 & $0.9 \pm 0.5$ & 0.182 \\
\hline & Full-term & $2.9 \pm 1.1$ & & $0.7 \pm 0.2$ & & $0.6 \pm 0.4$ & \\
\hline \multirow[t]{2}{*}{ Mediolateral } & Preterm & $7.7 \pm 4.4$ & 0.113 & $2.0 \pm 1.7$ & 0.211 & $1.2 \pm 0.6$ & 0.065 \\
\hline & Full-term & $4.5 \pm 1.9$ & & $1.1 \pm 0.5$ & & $0.7 \pm 0.4$ & \\
\hline \multirow[t]{2}{*}{$3 \mathrm{D}$} & Preterm & $9.2 \pm 4.1$ & 0.017 & $1.8 \pm 0.8$ & 0.028 & $1.4 \pm 0.8$ & 0.013 \\
\hline & Full-term & $4.8 \pm 0.9$ & & $1.0 \pm 0.3$ & & $0.8 \pm 0.4$ & \\
\hline
\end{tabular}

posterior and mediolateral) and for the resulting COM motion in the space (3D).

\section{Statistical analysis}

The data were processed with the Statistica 10.0 software (Stat-Soft, Inc., Tulsa, OK, USA). Differences between the two groups (COM range of motion, variability and velocity) were compared using the nonparametric Mann-Whitney U test.

\section{RESULTS}

Basic statistical characteristics of the measured parameters are shown in Table 2. The range of motion of the COM was significantly greater in preterm babies than in full-term babies, both in space $(9.2 \% / 4.8 \%)$ and in the craniocaudal (10.0\%/4.8\%) and anteroposterior $(5.3 \% / 2.9 \%)$ directions (Fig. 1).

The variability of the COM motion was significantly greater in the craniocaudal and anteroposterior directions in preterm babies than in full-term babies. This was also valid for the resulting motion of the COM in space (craniocaudal direction $2.0 \% / 1.0 \%$, anteroposterior direction $1.1 \% / 0.7 \%$ and motion in the space $1.8 \% / 1.0 \%$ ).

The velocity of the COM was significantly higher in preterm babies in the craniocaudal direction $(1.7 \% / 0.8 \%)$ and in the space $(1.4 \% / 0.8 \%)$; in the mediolateral direction. The $P$-value was close to the level of significance (0.05).

\section{DISCUSSION}

Kinematic analysis of movement is rarely used in the neonatal age. The published studies used different methods and assessed different parameters and movement patterns. Relatively frequent are studies analyzing spontaneous early kicking movements of newborns and young infants $^{12-16}$. In this area, however, no typical changes were found that would signal abnormal motor development ${ }^{17}$. This is not surprising given that newborns and young infants perform so-called holokinetic or generalized movements. Analyzing isolated movements of their limbs may not be appropriate for movement assessment. Moreover, spontaneous kicking movements are likely to be automa-

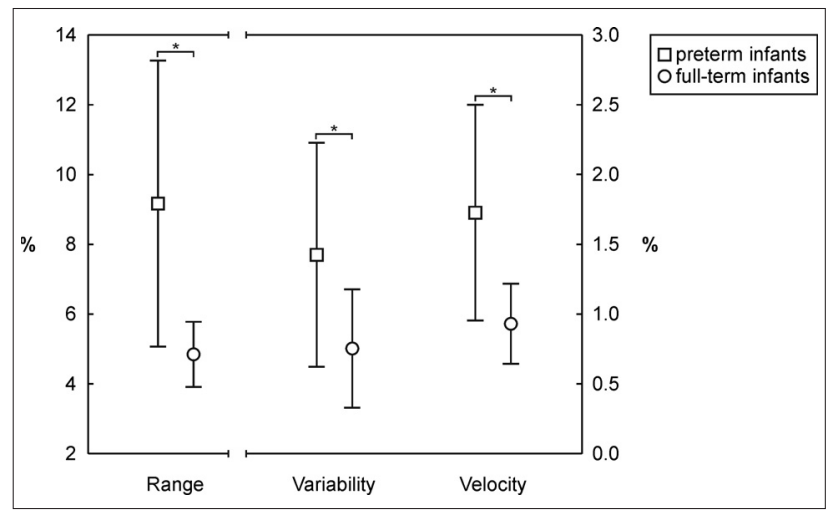

Fig. 1. Graphical comparison of observed groups $-3 D$ values. ${ }^{*} P<0.05$

tisms generated in the spinal cord and not in the higher nervous centers. Thus, abnormalities in spontaneous leg movements are unlikely to reflect the status of higher motor centers, abnormalities of which are responsible for motor development disorders.

To the best of our knowledge, kinematic analysis to assess the range of motion of the COM in newborns has not been reported so far. However, studies have been carried out that used force plates to measure the center of pressure (COP, point of the ground reaction force vector) and changes in its position in time $\mathrm{e}^{5,18-21}$. Darsaklis et al. ${ }^{5} \mathrm{com}$ pared characteristics of changes in the COP in preterm and full-term infants at corrected ages. The former were found to have significantly greater range of motion of the COP and its fluctuations in the craniocaudal direction. When assessing the complexity in movements, this group was found to have stereotypic movements compared with more complex changes in full-term infants. Other studies investigated the COP behaviour during infants' voluntary activity (voluntary reaching) at the corrected ages of 4 and 6 months, once again by comparing populations of preterm and full-term babies; at the same time, kinematic analysis was used to assess the quality of reaching. In this case, preterm infants were found to have a lower total range of motion of the COP that was associated with a better quality of reaching. The significance of these findings is not completely clear but the authors suggest an association with different preterm infants' adaptation to the extrauterine environment ${ }^{20,21}$. 
This pilot study aimed at determining whether kinematic analysis could be efficiently used to assess movements in newborns. Equipment-wise, the method is relatively simple. It is our opinion that recordings made with two cameras are sufficient for this type of study; more cameras allow more accurate quantifications but at the cost of longer time spent on data processing ${ }^{22}$. In the recordings, points were manually marked to minimize the burden to infants, with respect to problems brought about by marking points directly on the skin or attaching markers to the skin with adhesives. Ours is not an optimal solution due to potential inaccuracies when marking the points in movement recordings and, in particular, timeconsuming processing of recordings. Therefore, adaptations should be made to the method so that recordings are processed automatically.

The ascertained differences in COM movement in both studied groups may stem from the different environment in which preterm babies develop (effect of gravitation, forced position and need for full use of muscles to ensure breathing movements), length of adaptation to the external environment (postnatal age) as well as factors directly affecting motor control at both central and peripheral levels (severe health complications). This will have to be verified by further studies focusing on selected phases of ontogenesis.

Our findings suggesting differences in the movement of the COM between groups cannot be generalized due to either the small study group or its low homogeneity (differences in birth weight and gestational age, measurements not carried out at corrected ages, relatively short postnatal adaptation of full-term babies, etc.).

\section{CONCLUSION}

Kinematic analysis of movements using 3D videography may be used to investigate newborns' spontaneous movements. By determining the center of mass and its motion in time, neonatal postural activity may be indirectly assessed. The detected significant differences in certain characteristics of the motion of the COM in the group of preterm infants suggest the potential value of the method in early diagnosis of movement disorders in at-risk groups of newborns.

Acknowledgement: Supported by Palacky University Olomouc grant project FTK 2012:024.

Author contributions: JH, AM, LK: literature search; JH, AM: manuscript writing; JH, AM, MJ: study design; AM, JM, KH, NM: data collection; ZS, MJ: data analysis; JH, AM: data interpretation; ZS: statistical analysis, figures; JH, AM, MJ, LK: final approval.

Conflict of interest statement: None declared.

\section{REFERENCES}

1. Allen MC. Neurodevelopmental outcomes of preterm infants. Curr Opin Neurol 2008;21:123-8.

2. Kotagal S. Neurological examination of the newborn. In: UpToDate, Basow DS (Ed). UpToDate. Waltham, MA. (Accessed on February 02, 2014.)

3. Einspieler C, Prechtl HFR. Prechtl's assessment of general movements: A diagnostic tool for the functional assessment of the young nervous system. Ment Retard Dev D R 2005;11:61-7.

4. Einspieler C, Prechtl HFR, Ferrari F, Cioni G, Bos AF. The qualitative assessment of general movements in preterm, term and young infants - review of the methodology. Early Hum Dev 1997;50(1):47-60.

5. Darsaklis V, Snider LM, Majnemer A, Mazer B. Predictive validity of Prechtl's method on the qualitative assessment of general movements: a systematic review of the evidence. Dev Med Child Neurol 2011;53(10):896-906.

6. Harris SR. Movement analysis - an aid to early diagnosis of cerebral palsy. Phys Ther 1991;71(3):215-21.

7. Harris SR, Heriza CB. Measuring infant movement: Clinical and technological assessment techniques. Phys Ther 1987;67(12):1877-80.

8. Hadders-Algra M. Development of postural control during the first 18 months of life. Neural Plast 2004;12:99-107.

9. Dusing SC, Kyvelidou A, Mercer VS, Stergiou N. Infants born preterm exhibit different patterns of center-of-pressure movement than infants born at full term. Phys Ther 2009;89(12):1354-62.

10. Lafond D, Duarte M, Prince F. Comparison of three methods to estimate the center of mass during balance assessment. J Biomech 2004;37:1421-6.

11. Dempster WT, Gaughran GR. Properties of body segments based on size and weight. Am J Anat 1967;120(1):33-54.

12. Geerdink JJ, Hopkins B, Beek WJ, Heriza CB. The organization of leg movements in preterm and full-term infants after term age. Dev Psychobiol 1996;29(4):335-51.

13. Heriza CB. Organization of leg movements in preterm infants. Phys Ther 1988;68(9):1340-6.

14. Heriza CB. Comparison of leg movements in preterm infants at term with healthy full-term infants. Phys Ther 1988;68(11):1687-93.

15. Jeng SF, Chen LC, Yau KIT. Kinematic analysis of kicking movements in preterm infants with very low birth weight and full-term infants. Phys Ther 2002;82(2):148-59.

16. Landgraf JF, Carvalho RP, Tudella E. Method for the kinematic analysis of kicking movement in infants. Fisioter Pesqui [serial on the Internet]. 2013 Mar [cited 2014 Nov 10]; 20(1): 56-63. Available from: http://www.scielo.br/scielo.php?script=sci_arttext\&pid=S180929502013000100010\&lng=en.

17. van der Heide JC, Paolicelli PB, Boldrini A, Cioni G. Kinematic and qualitative analysis of lower-extremity movements in preterm infants with brain lesions. Phys Ther 1999;79(6):546-57.

18. Fallang $B$, Hadders-Algra M. Postural behavior in children born preterm. Neural Plast 2005;12(2-3):175-82.

19. Fallang B, Saugstad OD, Grogaard J, Hadders-Algra M. Kinematic quality of reaching movements in preterm infants. Pediatr Res 2003;53(5):836-42.

20. Fallang B, Saugstad OD, Hadders-Algra AM. Postural adjustments in preterm infants at 4 and 6 months post-term during voluntary reaching in supine position. Pediatr Res 2003;54(6):826-33.

21. Fallang B, Oien I, Hellem E, Saugstad OD et al. Quality of reaching and postural control in young preterm infants is related to neuromotor outcome at 6 years. Pediatr Res 2005;58(2):347-53.

22. Lima CD, Carvalho RP, Barros RML, Tudella E. Two different methods for kinematic analysis of head movements relating to eye-head coordination in infants. Rev bras fisioter [serial on the Internet]. 2008 Oct [cited 2014 Nov 15]; 12(5):425-31. Available from: http://www.scielo.br/scielo.php?script=sci_arttext \&pid=S141335552008000500013\&lng=en. 